\title{
A MIXED PARSEVAL-PLANCHEREL FORMULA
}

\section{OMRAN KOUBA}

Abstract. In this note, a general formula is proved. It expresses the integral on the line of the product of a function $f$ and a periodic function $g$ in terms of the Fourier transform of $f$ and the Fourier coefficients of $g$. This allows the evaluation of some oscillatory integrals.

Mathematics subject classification (2010): 42A16, 42A38, 42B20.

Keywords and phrases: Fourier series, Fourier transform, oscillatory integrals.

\section{REFERENCES}

[1] A. ERdÉlyi et AL., Tables of Integral Transforms, vol. I, McGraw Hill, New York, 1954.

[2] Y. KatZnelson, An Introduction to Harmonic Analysis, 3rd ed., Cambridge University Press, 2004.

[3] O. KoubA, Exact Evaluation of Some Highly Oscillatory Integrals, Journal of Classical Analysis 3, 1 (2013), 45-57. http://files.ele-math.com/articles/jca-03-04.pdf.

[4] G.P. Tolstov and R.A. Silverman, Fourier Series, Dover Books on Mathematics, Dover Publication, Inc., New York, 1962. 
[5] S. Tsipelis, Proposed problem V4-6, The Asymmetry Online Mathematical Journal 4 November (2013). http://www . asymmetry.gr/images/asymmetry/Asymmetry_V4_Nov_2013.pdf.

[6] J. WALDVOGEL, Towards a general error theory of the trapezoidal rule, Approximation and Computation, In honor of Gradimir V. Milovanović. W. Gautschi, G. Mastroianni, Th.M. Rassias (eds.), Springer Optimization and its Applications 42, Springer, New York, 2011, pp. 267-282.

[7] E. T. Whittaker and G. N. Watson, A Course of Modern Analysis, 4th ed., Cambridge Mathematical Library, Cambridge University Press, 1927 (reissued 1996). 